Chinese Journal of Organic Chemistry

NOTE

\title{
$\mathrm{pH}$ 调控下基于分子内电荷转移(ICT)机理的硫化氢荧光探针
}

\author{
朱继华 ${ }^{a, b}$ 张 浩 ${ }^{a}$ 刘 敏 ${ }^{a}$ 刘旌江 ${ }^{a}$ 廖 原 ${ }^{a}$ \\ 权正军*, $a$ 王喜存*,a \\ ( ${ }^{a}$ 西北师范大学化学化工学院 兰州 730070) \\ $\left({ }^{b}\right.$ 陇东学院化学化学工院 甘肃庆阳 745000)
}

\begin{abstract}
摘要 硫化氢 $\left(\mathrm{H}_{2} \mathrm{~S}\right)$ 和 $\mathrm{pH}$ 在环境和细胞内的许多过程中起着至关重要的作用. 为了更好地研究其功能, 需要一种快速、 灵敏地检测 $\mathrm{H}_{2} \mathrm{~S}$ 和 $\mathrm{pH}$ 的苂光探针. 设计合成了以萗二酰亚胺为荧光基团, 叠氮为识别位点, 基于分子内电荷转移(ICT) 机制的反应型苂光探针 $\mathbf{L}$. 该探针 $\mathbf{L}$ 在较宽的 $\mathrm{pH}$ 范围(4 11)内稳定性好, 对 $\mathrm{H}_{2} \mathrm{~S}$ 的选择性高, 响应速度快 $(3 \mathrm{~min})$, 检 测限 $\left(1.18 \mu \mathrm{mol} \cdot \mathrm{L}^{-1}\right)$ 较低, 在 $0 \sim 20 \mu \mathrm{mol} \cdot \mathrm{L}^{-1}$ 的范围内, 荧光强度与 $\mathrm{H}_{2} \mathrm{~S}$ 浓度呈良好的线性关系 $\left(R^{2}=0.99823\right)$. 此外, 当体系中存在 30 倍的 $\mathrm{H}_{2} \mathrm{~S}$ 时, 在 $2 \leqslant \mathrm{pH} \leqslant 6.5$ 的范围内, $\mathbf{L}$ 对 $\mathrm{pH}$ 变化显示线性关系 $\left(R^{2}=0.98764\right)$, 可用作 $\mathrm{pH}$ 探针. 该 探针在检测 $\mathrm{H}_{2} \mathrm{~S}$ 和 30 倍 $\mathrm{H}_{2} \mathrm{~S}$ 存在条件下研究细胞微环境 $\mathrm{pH}$ 变化的方法, 在分析检测和病理分析等方面具有潜在的应 用前景.
\end{abstract}

关键词＼cjkstart硫化氢; $\mathrm{pH}$; 苂光探针; 理论计算; ICT 机制

\section{An Intramolecular Charge Transfer (ICT)-Based Fluorescent Probe of Hydrogen Sulphide under pH Control Strategy}

\author{
Zhu, Jihua ${ }^{a, b}$ \\ Zhang, $\mathrm{Hao}^{a}$ \\ Liu, $\operatorname{Min}^{a}$ \\ Liu, Jingjiang ${ }^{a}$ \\ Liao, Yuan ${ }^{a}$ \\ Quan, Zhengjun ${ }^{*, a} \quad$ Wang, Xicun ${ }^{*, a}$ \\ ( ${ }^{a}$ College of Chemistry and Chemical Engineering, Northwest Normal University, Lanzhou 730070) \\ ( ${ }^{b}$ College of Chemistry and Chemical Engineering, Longdong University, Qingyang, Gansu 745000)
}

\begin{abstract}
Hydrogen sulfide $\left(\mathrm{H}_{2} \mathrm{~S}\right)$ and $\mathrm{pH}$ play a most important role in vivo and environment. A fluorescent probe for fast and sensitive detection of $\mathrm{H}_{2} \mathrm{~S}$ and $\mathrm{pH}$ are of vital importance for research their functions. A naphthalimide-based fluorescent probe $\mathbf{L}$ has been designed and synthesized based on intramolecular charge transfer (ICT) mechanism by utilizing azide as recognition site. The probe $\mathbf{L}$ has demonstrated good stability in the $\mathrm{pH}$ range of $4 \sim 11$. It showed satisfactory sensitivity and rapidly response (less than $3 \mathrm{~min})$ to $\mathrm{H}_{2} \mathrm{~S}$. An excellent linear relationship $\left(R^{2}=0.99823\right)$ displayed in the range of $\mathrm{H}_{2} \mathrm{~S}$ concentrations from $0 \mu \mathrm{mol} \cdot \mathrm{L}^{-1}$ to $20 \mu \mathrm{mol} \cdot \mathrm{L}^{-1}$ and the detection limit was $1.18 \mu \mathrm{mol} \cdot \mathrm{L}^{-1}$. Furthermore, in the present 30 equiv. $\mathrm{H}_{2} \mathrm{~S}$, it was successfully applied to detection of $\mathrm{pH}$ with good linear relationship $\left(R^{2}=0.98764\right)$ in the $\mathrm{pH}$ range of $2 \sim$ 6.5. This methods provide a reference for detection of $\mathrm{H}_{2} \mathrm{~S}$ and $\mathrm{pH}$ value in the presence of 30 equiv. $\mathrm{H}_{2} \mathrm{~S}$, which have potential application prospects in analytical detection and pathological analysis.
\end{abstract}

Keywords hydrogen sulfide; $\mathrm{pH}$; fluorescent probe; theoretical calculations; ICT mechanism

硫化氢 $\left(\mathrm{H}_{2} \mathrm{~S}\right)$ 是一种剧毒、具有 “臭鸡蛋” 气味的环 境危害气体, 其毒性是一氧化碳的 5 倍 ${ }^{[1]}$. 此外, $\mathrm{H}_{2} \mathrm{~S}$ 是 一种新型的具有细胞保护作用的信号分子, 与活性氧 (ROS) 和一氧化氮(NO)等其他活性分子一起被认为是一 种关键的信号分子 ${ }^{[2 \sim 4]}$. 在含毫摩尔浓度 $\mathrm{H}_{2} \mathrm{~S}$ 的低氧环
境中, 它在某些系统中起着电子供体和能源的作用; 在 低浓度或低释放速率下，具有有益的细胞保护作用，而 在高浓度或快速释放速率下, 具有明显的细胞毒性 ${ }^{[5]}$, 其通过影响细胞信号传导途径和靶蛋白的硫化作用, 调 节多种生理过程，包括心血管、神经传递、免疫反应、

\footnotetext{
* Corresponding authors. E-mail: quanzhengjun@hotmail.com; wangxicun@nwnu.edu.cn

Received October 20, 2019; revised November 12, 2019; published online December 11, 2019.

Project supported by the Longyuan Youth Innovative and Entrepreneurial Talents Project (No. 2019-39) and the Research Project of Higher Education in Gansu Province (No. 2018A-004).

陇原青年创新创业人才工程(No. 2019-39)和甘肃省高等学校科研(No. 2018A-004)资助项目.
} 
呼吸、胃肠道、肝脏、内分泌系统和循环系统 ${ }^{[6 ~ 9]}$. 此 外, 硫化氢的水平异常与一系列疾病有关, 包括糖尿 病、高血压、中风和阿尔茨海默症等 ${ }^{[10 \sim 12]}$. 因此, 发展 高选择性和高灵敏度检测 $\mathrm{H}_{2} \mathrm{~S}$ 的方法成为当前研究的 热点之一.

近年来, 很多用于 $\mathrm{H}_{2} \mathrm{~S}$ 检测的苂光探针被报

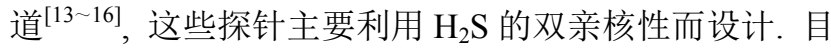
前报道的主要有对叠氮化物和硝基化合物的还原、对二 硝基苯基醚的硫解、对不饱和双键的加成和对铜等过渡 金属离子的配合等类型 ${ }^{[17 ~ 21]}$. 以分子间弱的作用力作 为识别位点的金属配合物探针具有响应速度快、灵敏度 高等优点, 但是对 $\mathrm{H}_{2} \mathrm{~S}$ 的选择性较低, 容易受到细胞中 其他含硫物种的干扰 ${ }^{[22]}$. 利用 $\mathrm{H}_{2} \mathrm{~S}$ 的亲核性和还原性设 计的反应型探针虽然可以实现硫化氢的高选择性识 别 ${ }^{[23]}$, 但是该类荧光探针也存在响应时间长 $(10 \sim 120$ min)、对内源性硫化氢的监测灵敏度不高等缺点而难以 实现 $\mathrm{H}_{2} \mathrm{~S}$ 的快速跟踪和实时检测 ${ }^{[24 ~ 27]}$. 因此, 寻找对 $\mathrm{H}_{2} \mathrm{~S}$ 具有更高的灵敏度、更短响应时间和分析物特异性 的检测方法仍然是一项具有吸引力和挑战性的工作.

细胞内特异性的酸碱度, 在细胞增殖、侵袭转移、 调亡、耐药性、离子转运等多种生理过程中起着至关重 要的作用, 特别是对癌细胞及其微环境中 $\mathrm{pH}$ 异常的检 测, 有助于研究细胞功能和病理过程, 有助于发现新的 $\mathrm{pH}$ 相关疾病的治疗方法 ${ }^{[28 ~ 30]}$. 因此, 设计合成一种既 能识别 $\mathrm{H}_{2} \mathrm{~S}$ 又能检测 $\mathrm{pH}$ 的多用途苂光探针更有意义. 鉴于此, 本文以光学稳定、苂光量子产率高的萗二酰亚 胺为苂光才, 利用 $\mathrm{H}_{2} \mathrm{~S}$ 对叠氮特异性的还原能力而设计 合成了一种 $\mathrm{pH}$ 调控下用于 $\mathrm{H}_{2} \mathrm{~S}$ 检测的基于分子内电荷 转移(ICT)机制的荧光探针 $\mathbf{L}$, 该探针可以实现对 $\mathrm{H}_{2} \mathrm{~S}$ 的 荧光增强响应, 并且选择性好、响应速度快. 此外, 利用 不同 $\mathrm{pH}$ 环境下 $\mathrm{H}_{2} \mathrm{~S}$ 和探针 $\mathbf{L}$ 作用苂光强度的变化, 可 以对酸性环境下的 $\mathrm{pH}$ 进行检测.

\section{1 结果与讨论}

\section{1 荧光探针 $L$ 的设计合成}

本文以 4-溴-1,8-䒺二酸酐和炔丙胺为原料, 反应得 到中间体 3, 然后和叠氮化钠在室温下即可方便的得到 苂光探针 $\mathbf{L}$. 探针 $\mathbf{L}$ 结构经红外光谱、核磁和 HRMS 进 行了鉴定. 如图 1 所示, 探针 $\mathbf{L}$ 的最大吸收峰为 $365 \mathrm{~nm}$, 加入 50 倍的 $\mathrm{H}_{2} \mathrm{~S}$ 后, 该吸收峰强度减弱, $459 \mathrm{~nm}$ 处出现 了新的 $\pi \rightarrow \pi *$ 跃迁吸收峰, 吸收峰的红移说明生成物分 子内跃迁所需能量减小, 该现象可能为萗二酰亚胺和叠 氮之间的分子内电荷转移(ICT)过程被消除所致 ${ }^{[31]}$, 最 大发射峰位于 $539 \mathrm{~nm}$, 斯托克位移为 $174 \mathrm{~nm}$. 因此, 探 针 $\mathbf{L}$ 在使用过程中受背景荧光的干扰较小. 在没有 $\mathrm{H}_{2} \mathrm{~S}$

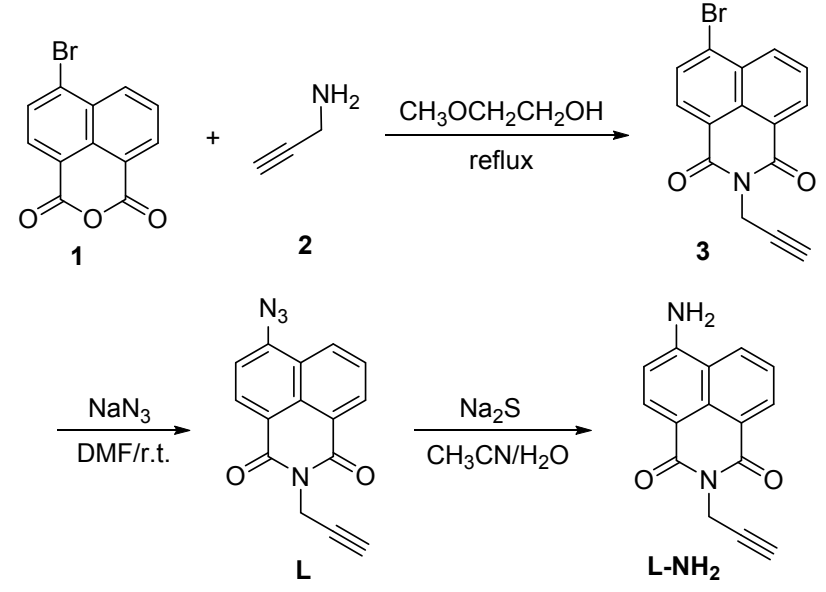

图式 1 荧光探针 $\mathbf{L}$ 的合成及对 $\mathrm{H}_{2} \mathrm{~S}$ 可能的识别机制 Scheme 1 Synthetic fluorescent probe $\mathbf{L}$ and proposed detection mechanism with $\mathrm{H}_{2} \mathrm{~S}$.

的情况下, 在 $\mathrm{CH}_{3} \mathrm{CN} / \mathrm{PBS}(V: V=1: 1)$ 混合物溶剂中, 探针 $\mathbf{L}$ 呈现很微弱的苂光, 在加入 30 equiv. $\mathrm{H}_{2} \mathrm{~S}$ 后, 随 着反应的进行, 黄绿色苂光逐渐增强, 在 $365 \mathrm{~nm}$ 紫外灯 照射下，探针 $\mathbf{L}$ 可以用于 $\mathrm{H}_{2} \mathrm{~S}$ 的裸眼识别. 另外，探针 $\mathbf{L}$ 分子中引入炔基，可以和烯烃等通过聚合反应得到功 能化的高分子材料, 为进一步拓展荧光探针分子的设计 策略提供有价值的参考.

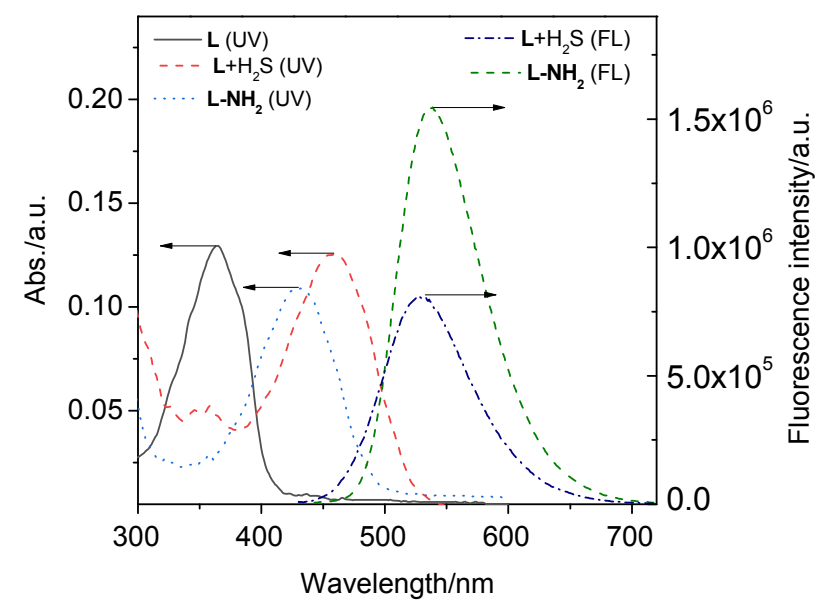

图 1 在 $\mathrm{CH}_{3} \mathrm{CN} / \mathrm{PBS}\left(V: V=1: 1,20 \mathrm{mmol} \cdot \mathrm{L}^{-1}, \mathrm{pH}=7.4\right)$ 的 缓冲体系中测试 $\mathbf{L} 、 \mathbf{L}+\mathrm{H}_{2} \mathrm{~S}\left(200 \mu \mathrm{mol} \cdot \mathrm{L}^{-1}\right)$ 和 $\mathbf{L}-\mathbf{N H}_{\mathbf{2}}$ 的紫外 吸收光谱 $\left(10 \mu \mathrm{mol} \cdot \mathrm{L}^{-1}\right)$ 和苂光光谱 $\left(1 \mu \mathrm{mol} \cdot \mathrm{L}^{-1}\right)\left(\lambda_{\mathrm{ex}}=420 \mathrm{~nm}\right)$ Figure $1 \mathrm{UV}$ absorption $\left(10 \mu \mathrm{mol} \cdot \mathrm{L}^{-1}\right)$ and fluorescence intensity $\left(1 \mu \mathrm{mol} \cdot \mathrm{L}^{-1}\right)$ of probe $\mathbf{L}, \mathbf{L}+\mathrm{H}_{2} \mathrm{~S}\left(200 \mu \mathrm{mol} \cdot \mathrm{L}^{-1}\right)$ and L-NH $\mathbf{N H}_{2}$ in $\mathrm{CH}_{3} \mathrm{CN} / \mathrm{PBS}$ buffer $\left(V: V=1: 1,20 \mathrm{mmol} \cdot \mathrm{L}^{-1}, \mathrm{pH}=\right.$ 7.4) solution $\left(\lambda_{\mathrm{ex}}=420 \mathrm{~nm}\right)$

\section{2 荧光探针 $L$ 的选择性和干扰}

苂光探针 $\mathbf{L}$ 的选择性作为我们所期望的最重要的 特征. 为了更好地研究其特异选择性, 在实验过程中, 常见阴、阳离子和氨基酸等不同被检测物的浓度 (50 
$\mu \mathrm{mol} \cdot \mathrm{L}^{-1}$ )为探针 $\mathbf{L}$ 浓度的 50 倍. 如图 2 和图 3 所示, 探 针 $\mathbf{L}$ 在 $539 \mathrm{~nm}$ 处的荧光强度与未加入干扰和检测物的 空白样品比较, 只有加入 $\mathrm{H}_{2} \mathrm{~S}$ 的体系苂光强度呈现大幅 度的增强, 而其它氨基酸、无机离子对探针 $\mathbf{L}$ 的苂光强 度几乎没有明显影响. 因此, 探针 $\mathbf{L}$ 可以高选择性地检 测 $\mathrm{H}_{2} \mathrm{~S}$. 此外, 研究了探针 $\mathbf{L}$ 在 $100 \mu \mathrm{mol} \cdot \mathrm{L}^{-1}$ 不同干扰 物存在条件下对 $\mathrm{H}_{2} \mathrm{~S}$ 的苂光响应. 探针 $\mathbf{L}$ 对 $\mathrm{H}_{2} \mathrm{~S}$ 的检测 几乎不受其他检测物的影响, 只有次氯酸根离子 $\left(\mathrm{ClO}^{-}\right)$ 加入后再加入 $\mathrm{H}_{2} \mathrm{~S}$, 荧光强度几乎没有变化, 说明 $\mathrm{ClO}^{-}$ 对 $\mathrm{H}_{2} \mathrm{~S}$ 的识别具有明显的干扰作用. 其主要原因如下: (1) $\mathrm{H}_{2} \mathrm{~S}$ 具有还原性, 而 $\mathrm{HClO}$ 具有强的氧化性, 在溶液 中二者因发生氧化还原反应而消耗 $\mathrm{H}_{2} \mathrm{~S}$; (2) 即使有微量 的 $\mathrm{H}_{2} \mathrm{~S}$ 和 $\mathbf{L}$ 能够竞争反应得到氨基, 而氨基也容易被氧 化成硝基而猝灭荧光探针的荧光.

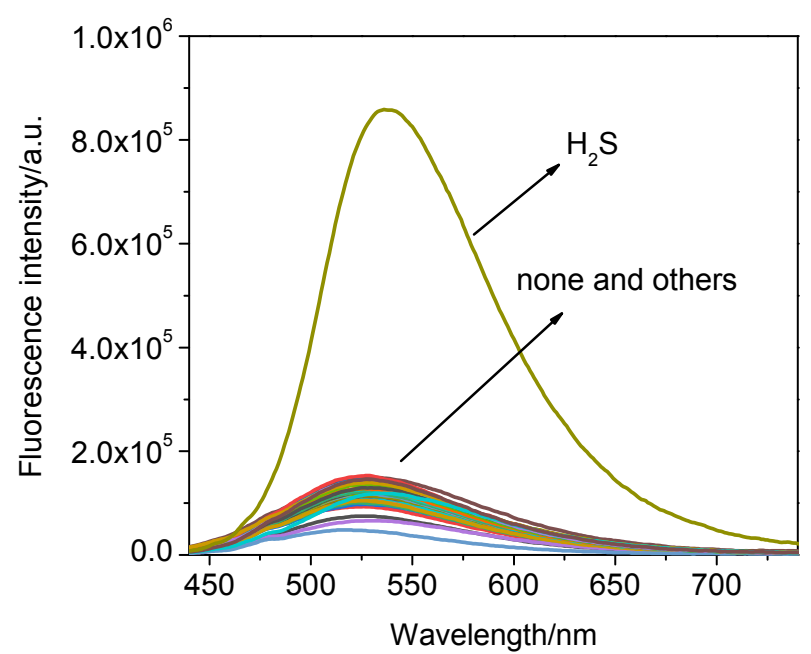

图 2 苂光探针 $\mathbf{L}\left(1 \mu \mathrm{mol} \cdot \mathrm{L}^{-1}\right)$ 对不同物质 $\left(50 \mu \mathrm{mol} \cdot \mathrm{L}^{-1}\right)$ 的苂 光响应 $\left(\lambda_{\mathrm{ex}}=420 \mathrm{~nm}\right)$

Figure 2 Fluorescence responses of probe $\mathbf{L}\left(1 \mu \mathrm{mol} \cdot \mathrm{L}^{-1}\right)$ to various substances $\left(50 \mu \mathrm{mol} \cdot \mathrm{L}^{-1}\right)\left(\lambda_{\mathrm{ex}}=420 \mathrm{~nm}\right)$

\section{3 荧光探针 $L$ 的响应时间}

$\mathrm{H}_{2} \mathrm{~S}$ 在体内分解代谢很快, 荧光探针的响应速度是 评价探针性能的一个关键因素, 因此对探针 $\mathbf{L}$ 进行了动 力学研究. 结果如图 4 所示, 探针 $\mathbf{L}\left(1 \mu \mathrm{mol} \cdot \mathrm{L}^{-1}\right)$ 的体系 中加入 30 equiv. $\mathrm{H}_{2} \mathrm{~S}$ 后, $539 \mathrm{~nm}$ 处的苂光强度迅速增强, 随着反应的进行, 苂光强度增加速率逐渐变小, $3 \mathrm{~min}$ 后 苂光强度基本保持不变, 结果表明探针 $\mathbf{L}$ 可以快速检测 $\mathrm{H}_{2} \mathrm{~S}$.

\section{4 荧光探针 $\mathrm{L}$ 的 $\mathrm{pH}$ 响应}

从图 5 可以看出, 苂光探针 $\mathbf{L}$ 在 $4 \leqslant \mathrm{pH} \leqslant 11$ 的范围 内在 $539 \mathrm{~nm}$ 处的荧光强度基本保持不变, 说明 $\mathbf{L}$ 具有 很好的酸碱耐受性, 可以在广泛 $\mathrm{pH}$ 范围内应用. 在 30 equiv. $\mathrm{H}_{2} \mathrm{~S}$ 存在条件下 (如图 6 所示), 在 $2 \leqslant \mathrm{pH} \leqslant 6.5$ 的 范围内, 随着 $\mathrm{pH}$ 值的减小, $\mathbf{L}$ 荧光强度逐渐减弱并呈现

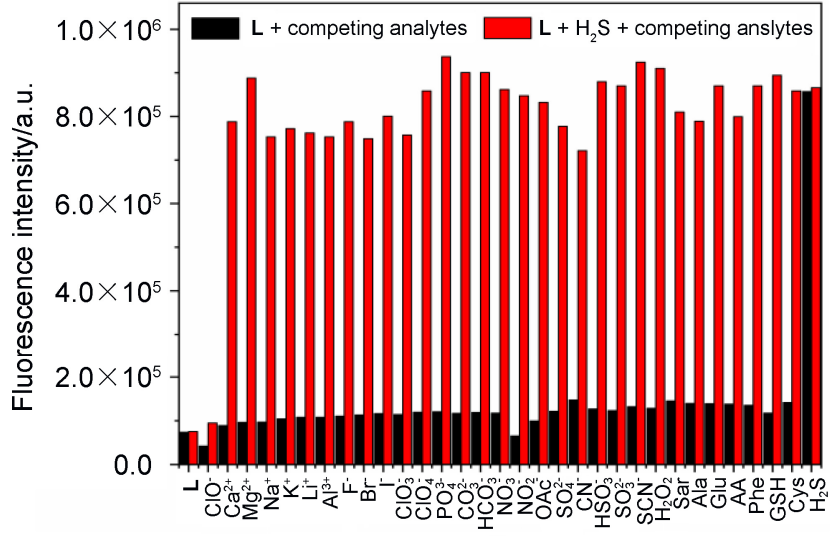

图 3 苂光探针 $\mathbf{L}\left(1 \mu \mu \mathrm{mol} \cdot \mathrm{L}^{-1}\right)$ 分别加入不同分析物 (50 $\left.\mu \mathrm{mol} \cdot \mathrm{L}^{-1}\right)$ 后的荧光强度(黑色), 以及在干扰物存在条件下加 入 $\mathrm{H}_{2} \mathrm{~S}\left(50 \mu \mathrm{mol} \cdot \mathrm{L}^{-1}\right)$ 后体系的苂光强度(红色) $\left(\lambda_{\mathrm{ex}}=420 \mathrm{~nm}\right.$, $\left.\lambda_{\mathrm{em}}=539 \mathrm{~nm}\right)$

Figure 3 Fluorescence intensity probe $\mathbf{L}\left(1 \mu \mathrm{mol} \cdot \mathrm{L}^{-1}\right)$ after addition of various analytes $\left(50 \mu \mathrm{mol} \cdot \mathrm{L}^{-1}\right)$ (black )and the fluorescence response to $\mathrm{H}_{2} \mathrm{~S}\left(50 \mu \mathrm{mol} \cdot \mathrm{L}^{-1}\right)$ in the presence of analytes $\left(50 \mu \mathrm{mol} \cdot \mathrm{L}^{-1}\right)(\mathrm{red})\left(\lambda_{\mathrm{ex}}=420 \mathrm{~nm}, \lambda_{\mathrm{em}}=539 \mathrm{~nm}\right)$



图4 苂光探针 $\mathbf{L}\left(1 \mu \mathrm{mol} \cdot \mathrm{L}^{-1}\right.$ 在加入 $\mathrm{H}_{2} \mathrm{~S}\left(50 \mu \mathrm{mol} \cdot \mathrm{L}^{-1}\right)$ 后荧光 强度随时间变化 $\left(\lambda_{\mathrm{ex}}=420 \mathrm{~nm}, \lambda_{\mathrm{em}}=539 \mathrm{~nm}\right)$

Figure 4 Fluorescence intensity of probe $\mathbf{L}\left(1 \mu \mathrm{mol} \cdot \mathrm{L}^{-1}\right)$ as a function of time after addition of $\mathrm{H}_{2} \mathrm{~S}\left(50 \mu \mathrm{mol} \cdot \mathrm{L}^{-1}\right)\left(\lambda_{\mathrm{ex}}=420\right.$ $\left.\mathrm{nm}, \lambda_{\mathrm{em}}=539 \mathrm{~nm}\right)$

良好的线性关系 $\left(R^{2}=0.98764\right)$, 说明荧光探针 $\mathbf{L}$ 在 $\mathrm{H}_{2} \mathrm{~S}$ 介导下可以对酸性的微弱变化产生明显响应, 可以用于 $\mathrm{pH}$ 检测. 该结果和文献报道的 $\mathrm{H}_{2} \mathrm{~S}$ 探针随 $\mathrm{pH}$ 变化有相 似的响应关系 ${ }^{[32,33]}$. 因此, 选择 $\mathrm{CH}_{3} \mathrm{CN} / \mathrm{PBS}(V: V=$ $1: 1,20 \mathrm{mmol} \cdot \mathrm{L}^{-1}, \mathrm{pH}=7.4$ ) 缓冲溶液作为 $\mathrm{H}_{2} \mathrm{~S}$ 识别的 测试体系, 以确保测试过程中探针 $\mathbf{L}$ 的苂光强度不会受 到 $\mathrm{pH}$ 微弱变化的影响.

\section{5 荧光探针 $L$ 对 $\mathrm{H}_{2} \mathrm{~S}$ 的荧光滴定}

荧光探针对待测物的定量分析是衡量其检测能力 的一个重要指标. 通过 $\mathrm{H}_{2} \mathrm{~S}$ 对探针 $\mathbf{L}$ 的苂光滴定实验, 


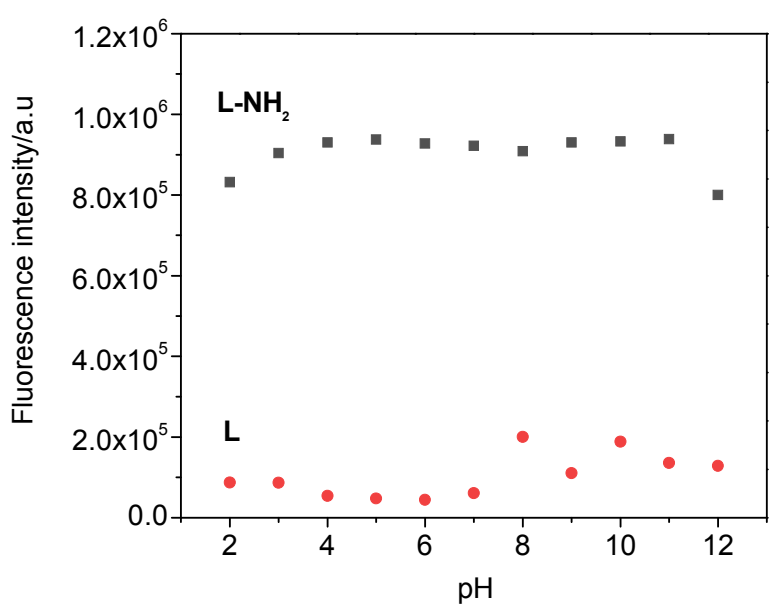

图 $5 \mathrm{pH}$ 对 $\mathbf{L}\left(1 \mu \mathrm{mol} \cdot \mathrm{L}^{-1}\right)$ 和 $\mathbf{L}-\mathbf{N H}_{2}\left(0.5 \mu \mathrm{mol} \cdot \mathrm{L}^{-1}\right)$ 苂光强度 的影响 $\left(\lambda_{\mathrm{ex}}=420 \mathrm{~nm}, \lambda_{\mathrm{em}}=539 \mathrm{~nm}, t=5 \mathrm{~min}\right)$

Figure 5 Fluorescence intensity of $\mathbf{L}\left(1 \mu \mathrm{mol} \cdot \mathrm{L}^{-1}\right)$ and $\mathbf{L}-\mathbf{N H}_{2}$ $\left(0.5 \mu \mathrm{mol} \cdot \mathrm{L}^{-1}\right)$ with different $\mathrm{pH}\left(\lambda_{\mathrm{ex}}=420 \mathrm{~nm}, \lambda_{\mathrm{em}}=539 \mathrm{~nm}, t=\right.$ $5 \mathrm{~min})$
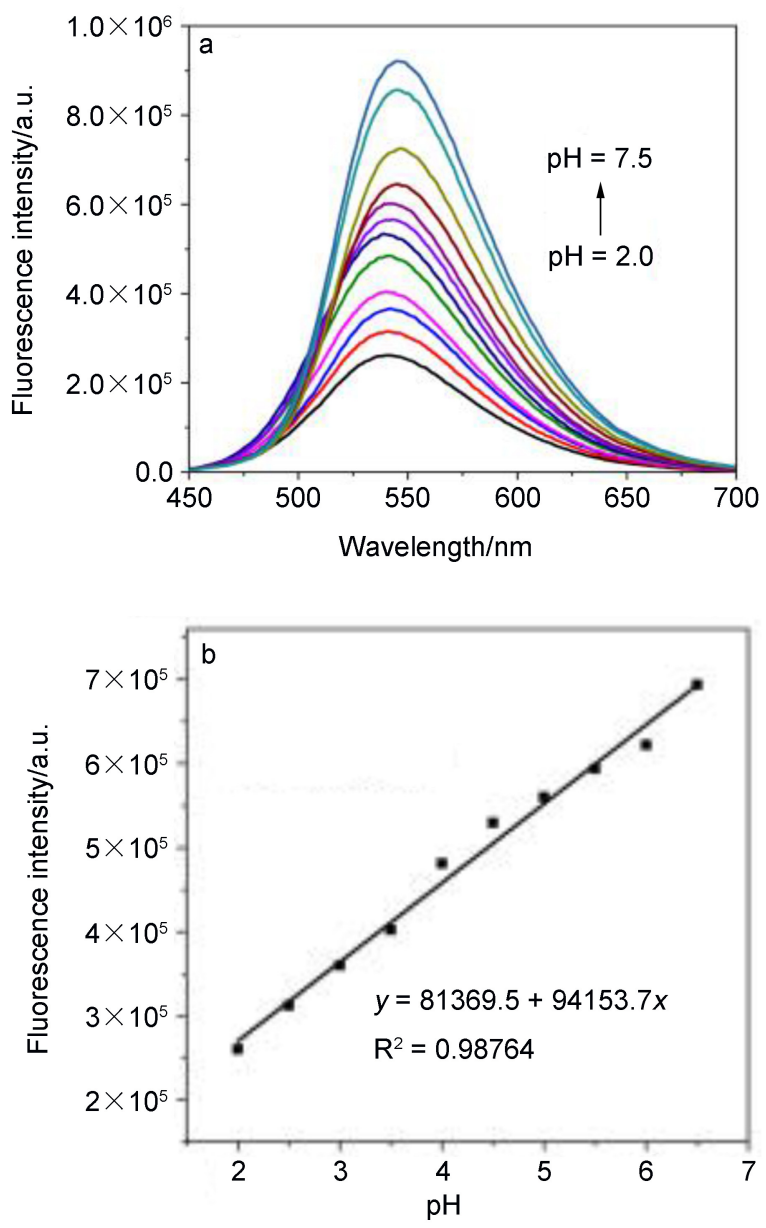

图 6 (a) 在 $\mathrm{H}_{2} \mathrm{~S}\left(30 \mu \mathrm{mol} \cdot \mathrm{L}^{-1}\right)$ 存在的条件下 $\mathbf{L}\left(1 \mu \mathrm{mol} \cdot \mathrm{L}^{-1}\right)$ 随 $\mathrm{pH}$ 的变化光强度, 以及(b) $\mathbf{L}$ 的荧光强度随 $\mathrm{pH}$ 值变化的线 性关系 $\left(\lambda_{\mathrm{ex}}=420 \mathrm{~nm}, \lambda_{\mathrm{em}}=539 \mathrm{~nm}\right)$

Figure 6 (a) Changes of the fluorescence spectra of $\mathbf{L}$ (1 $\left.\mu \mathrm{mol} \cdot \mathrm{L}^{-1}\right)$ in presence of $\mathrm{H}_{2} \mathrm{~S}\left(30 \mu \mathrm{mol} \cdot \mathrm{L}^{-1}\right)$ with increasing $\mathrm{pH}$ from 2.0 to 6.5 , and (b) plot of the fluorescence intensity of $\mathbf{L}$ with different $\mathrm{pH}\left(\lambda_{\mathrm{ex}}=420 \mathrm{~nm}, \lambda_{\mathrm{em}}=539 \mathrm{~nm}\right)$
研究了苂光探针的灵敏度和线性范围. 如图 7 所示, 逐 渐加入 20 equiv. $\mathrm{H}_{2} \mathrm{~S}$ 时, 荧光探针 $\mathbf{L}$ 在 $539 \mathrm{~nm}$ 处吸收 强度逐渐增强，在 $0 \sim 20 \mu \mathrm{mol} \cdot \mathrm{L}^{-1}$ 的范围内，苂光强度 与 $\mathrm{H}_{2} \mathrm{~S}$ 浓度呈线性关系 $\left(R^{2}=0.99823\right)$. 根据检测限 (LOD) 计算公式: $\mathrm{LOD}=3 \sigma / k$ (其中 $\sigma$ 为未添加 $\mathrm{H}_{2} \mathrm{~S}$ 时荧 光探针荧光光谱变化的标准偏差, $k$ 为直线斜率), 计算 得到探针 $\mathbf{L}$ 对 $\mathrm{H}_{2} \mathrm{~S}$ 的检测限为 $1.18 \mu \mathrm{mol} \cdot \mathrm{L}^{-1}$, 表明苂光 探针分子对 $\mathrm{H}_{2} \mathrm{~S}$ 具有较好的检测灵敏度.
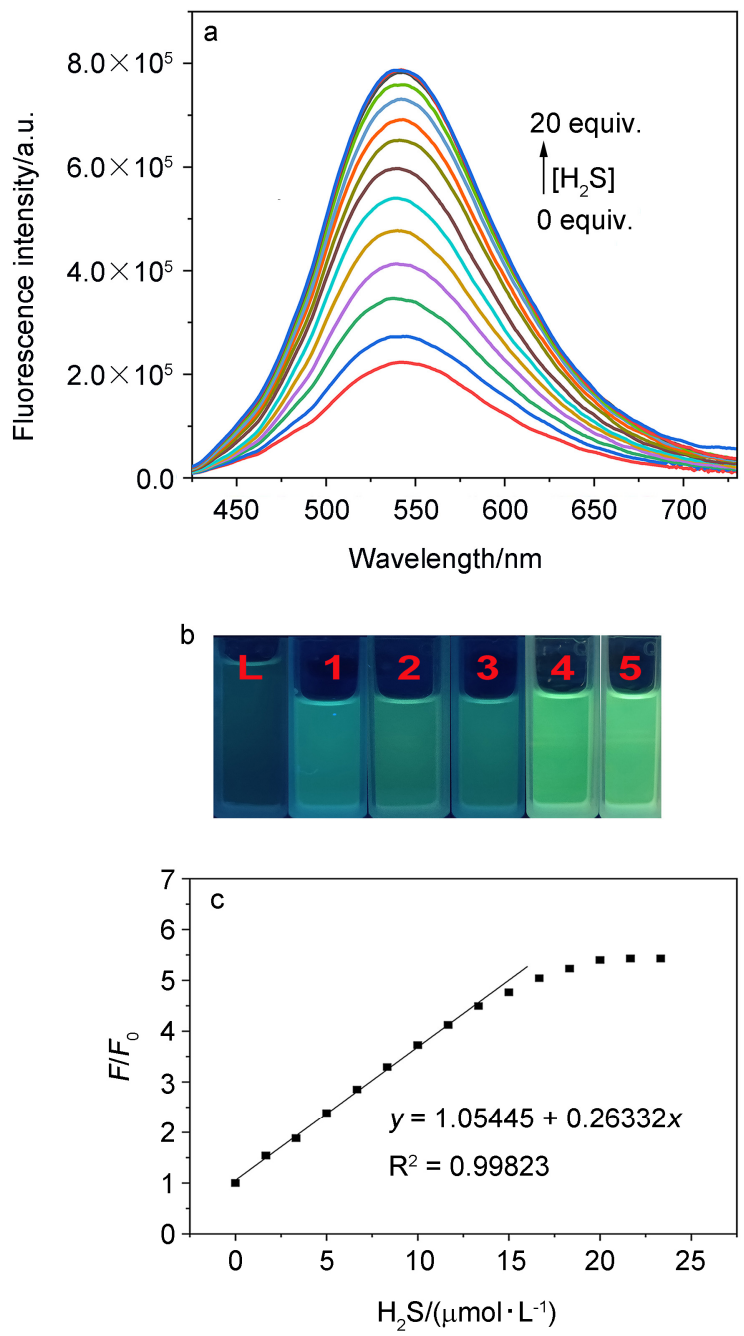

图 7 (a) $\mathbf{L}\left(1 \mu \mathrm{mol} \cdot \mathrm{L}^{-1}\right)$ 在不同浓度的 $\mathrm{H}_{2} \mathrm{~S}\left(0 \sim 25 \mu \mathrm{mol} \cdot \mathrm{L}^{-1}\right)$ 溶液中的荧光光谱, (b) $\mathbf{L}\left(10 \mu \mathrm{mol} \cdot \mathrm{L}^{-1}\right)$ 加入不同浓度的 $\mathrm{H}_{2} \mathrm{~S}$ $\left(1 \sim 5\right.$ 分别为 $10 、 20 、 30 、 50$ 和 $\left.100 \mu \mathrm{mol} \cdot \mathrm{L}^{-1}\right)$ 在 $365 \mathrm{~nm}$ 的紫 外灯照射下的照片, 以及(c) $\mathbf{L}$ 的荧光强度在 $539 \mathrm{~nm}$ 随 $\mathrm{H}_{2} \mathrm{~S}$ 浓 度 $\left(0 \sim 25 \mu \mathrm{mol} \cdot \mathrm{L}^{-1}\right)$ 变化的线性关系 $\left(\lambda_{\mathrm{ex}}=420 \mathrm{~nm}, \lambda_{\mathrm{em}}=539\right.$ $\mathrm{nm}, t=5 \mathrm{~min}$ )

Figure 7 (a) Fluorescence spectra of $\mathbf{L}\left(1 \mu \mathrm{mol} \cdot \mathrm{L}^{-1}\right)$ with different concentration of $\mathrm{H}_{2} \mathrm{~S}\left(0 \sim 25 \mu \mathrm{mol} \cdot \mathrm{L}^{-1}\right)$, (b) photograph of $\mathbf{L}\left(10 \mu \mathrm{mol} \cdot \mathrm{L}^{-1}\right)$ with different concentration of $\mathrm{H}_{2} \mathrm{~S}(1 \sim 5$ are $10,20,30,50$ and $100 \mu \mathrm{mol} \cdot \mathrm{L}^{-1}$, respectively) under $365 \mathrm{~nm}$ UV-light, and (c) plot of the fluorescence intensity of $\mathbf{L}$ with different concentration of $\mathrm{H}_{2} \mathrm{~S}\left(\lambda_{\mathrm{ex}}=420 \mathrm{~nm}, \lambda_{\mathrm{em}}=539 \mathrm{~nm}, t=5\right.$ $\min )$ 


\section{6 理论计算}

为了研究荧光探针 $\mathbf{L}$ 对 $\mathrm{H}_{2} \mathrm{~S}$ 的识别机制, 使用 Gaussian 09 程序, 采用 DFT/B3LYP/6-31G (d,p)基组进 行了理论分析. 优化获得能量最小的荧光探针 $\mathbf{L}$ 和 $\mathbf{L}-\mathrm{NH}_{2}$ 的结构. 为了进一步研究荧光探针 $\mathrm{L}$ 和 $\mathbf{L}-\mathrm{NH}_{2}$ 单重激发态的前线轨道能级, 采用同样基组进行了 TD-DFT 计算, 得到它们的前线轨道能量和分子跃迁能. 如图 8 所示, 探针 $\mathbf{L}$ 被激发后, 一个电子从 $\mathrm{HOMO}$ 轨道 跃迁至 $\mathrm{LUMO}$ 轨道 $(\mathrm{S} 1 \rightarrow \mathrm{S} 0$, 振子强度 $f=0.3279)$, 跃迁 能为 $3.67 \mathrm{eV}$, 轨道贡献率为 $97.4 \%$, 位于基态的叠氮基 团中的电子部分被转移到菜环部分, ICT 过程的 $\pi \rightarrow \pi^{*}$ 跃迁对应的吸收波长为 $368 \mathrm{~nm}$ (实验值为 $365 \mathrm{~nm}$ ). $\mathbf{L}-\mathrm{NH}_{2}$ 分子中 $\mathrm{HOMO}$ 和 $\mathrm{LUMO}$ 轨道的电子云在整个荧 光团上处于离域状态, 当电子从 $\mathrm{HOMO}$ 轨道跃迁至 LUMO 轨道时, 电子在 LUMO 和 HOMO 上未观察到明 显的重新分布, $\mathrm{S}_{1} \rightarrow \mathrm{S}_{0}(f=0.2014)$ 跃迁的贡献率为 $96.3 \%$, 跃迁能为 $3.57 \mathrm{eV}$. 在叠氮被 $\mathrm{H}_{2} \mathrm{~S}$ 还原为氨基的 过程中, 和萗环相连的氮原子 $(\mathrm{N} 12)$ 从缺电子 ( 0.014462)变成了富电子 $(0.009925) 、 \mathbf{L}-\mathbf{N H}_{2}$ 的吸收波长 红移(计算值 $387 \mathrm{~nm}$ )和菜二酰亚胺部分静电势增加都 说明环的电子云密度增加, $\pi \rightarrow \pi *$ 跃迁所需能量减小, 结 果表明探针 $\mathbf{L}$ 对 $\mathrm{H}_{2} \mathrm{~S}$ 的识别过程为 ICT 机制, 理论计算 与实验结果一致.
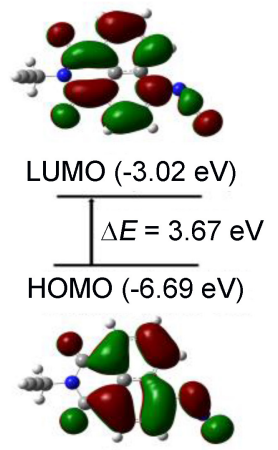

L
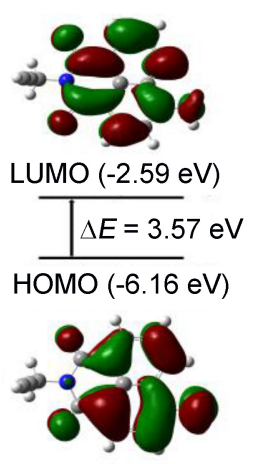

$\mathrm{L}-\mathrm{NH}_{2}$
图 $8 \mathrm{~L}$ 和 $\mathrm{L}_{-} \mathrm{NH}_{2}$ 的优化分子结构和激发态前线轨道能量

Figure 8 Optimized structures and frontier molecular orbital energy of of $\mathbf{L}$ and $\mathbf{L}-\mathbf{N H}_{2}$ at the excited state.

\section{7 荧光探针 $L$ 的识别机理}

苂光探针 $\mathbf{L}$ 与 $\mathrm{H}_{2} \mathrm{~S}$ 通过还原反应将䒺二酰亚胺 4 号位拉电子的叠氮还原为推电子的氨基, 使萗二酰亚胺 基团上的电子云密度增大, 从而实现对 $\mathrm{H}_{2} \mathrm{~S}$ 的识别. 为 了验证所提出的机制, 进行了量子化学计算、红外光谱、 高分辨质谱和核磁滴定分析. 红外光谱显示, $\mathbf{L}$ 在 $2120.0 \mathrm{~cm}^{-1}$ 出现氮氮三键的特征吸收峰, 而 $\mathbf{L}-\mathbf{N H}_{2}$ 红 外光谱中的三键消失, 在 3472.6 和 $3357.8 \mathrm{~cm}^{-1}$ 处出现
一对伯胺的特征吸收峰, 说明叠氮被 $\mathrm{H}_{2} \mathrm{~S}$ 还原成了氨 基. 在 $\mathbf{L}$ 与 $\mathrm{H}_{2} \mathrm{~S}$ 反应混合物的高分辨质谱中，对应 $\mathbf{L}$ 的 $[\mathrm{M}+\mathrm{Na}+\mathrm{H}]^{+} 300.0534$ 峰消失, 出现 1 个与 $\mathbf{L}-\mathbf{N H}_{2}$ (理 论值: 251.0815)一致的 $[\mathrm{M}+\mathrm{H}]^{+}$(251.0816)峰, 说明荧 光探针 $\mathbf{L}$ 和 $\mathrm{H}_{2} \mathrm{~S}$ 发生还原反应得到了化合物 $\mathbf{L}-\mathbf{N H}_{\mathbf{2}}$. 此 外, 核磁滴定结果如图 9 所示, 加入 10 equiv.的 $\mathrm{H}_{2} \mathrm{~S}$ 后, ${ }^{1} \mathrm{H}$ NMR 显示为两种化合物的混合物, 当增加 $\mathrm{H}_{2} \mathrm{~S}$ 的量 为 50 equiv. 时, ${ }^{1} \mathrm{H}$ NMR 谱仅有一种化合物的峰. 和 $\mathbf{L}$ 相 比, 加入 $\mathrm{H}_{2} \mathrm{~S}$ 后的 ${ }^{1} \mathrm{H}$ NMR 谱中的 $\mathrm{H}$ 原子信号峰向高场 移动, 说明拉电子的叠氮变成了推电子的氨基后, 䒬环 的电子云密度增加, 分子内的 ICT 过程消失. 为了探索 $\mathbf{L}$ 在 $\mathrm{H}_{2} \mathrm{~S}$ 存在条件下 $\mathrm{pH}$ 的响应机制, 测试了不同 $\mathrm{pH}$ 值 $\mathrm{CH}_{3} \mathrm{CN} / \mathrm{PBS}\left(V: V=1: 1,20 \mathrm{mmol} \cdot \mathrm{L}^{-1}\right)$ 体系中 $\mathbf{L}-\mathbf{N H}_{2}$ 在 $539 \mathrm{~nm}$ 处苂光强度. 如图 5 所示, 在 $\mathrm{pH}<4$ 的条件下, 荧光强度随 $\mathrm{pH}$ 值减小有微小的减弱; 在 $4<\mathrm{pH}<12$ 范 围内, 荧光强度基本保持不变, 说明 $\mathrm{pH}$ 变化不是和 $\mathbf{L}-\mathrm{NH}_{2}$ 相互作用影响荧光强度, 而是 $\mathrm{pH}$ 和 $\mathrm{H}_{2} \mathrm{~S}$ 的协同 作用控制 $\mathbf{L}-\mathbf{N H}_{\mathbf{2}}$ 的生成影响苂光强度.

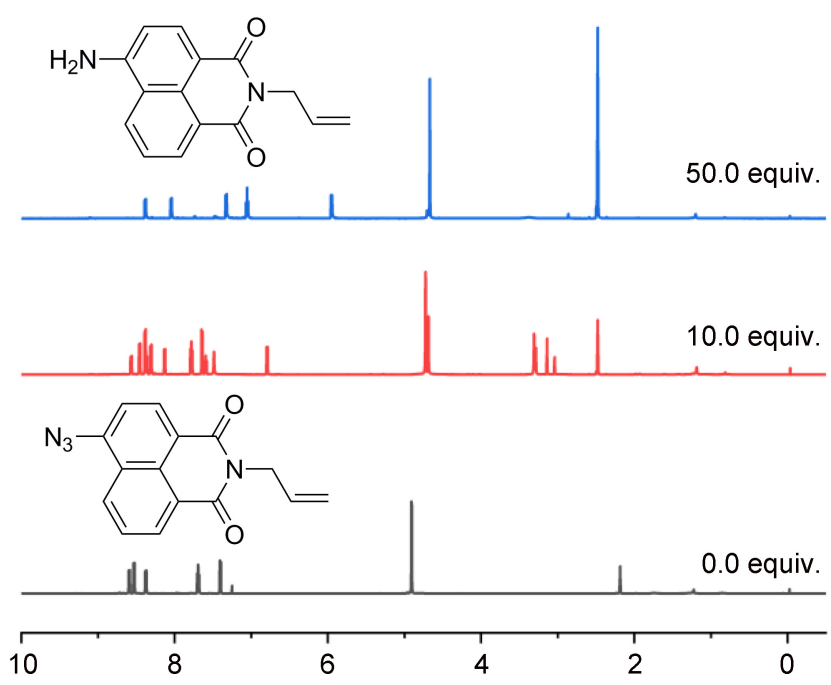

图 $9 \mathbf{L}$ 和 $\mathrm{H}_{2} \mathrm{~S}$ 的核磁滴定

Figure $9{ }^{1} \mathrm{H}$ NMR spectra of $\mathbf{L}$ with various equiv of $\mathrm{H}_{2} \mathrm{~S}$

\section{2 结论}

本文设计合成了一种基于菜二酰亚胺的化学反应 型 $\mathrm{H}_{2} \mathrm{~S}$ 苂光探针 $\mathbf{L}$. 我们通过红外光谱、核磁滴定和理 论计算对探针 $\mathbf{L}$ 的传感机制进行了分析. 结果表明: 探 针 $\mathbf{L}$ 苂光团上的叠氮被 $\mathrm{H}_{2} \mathrm{~S}$ 还原成氨基后，经过分子内 的电荷转移(ICT)过程, 可以实现 $\mathrm{H}_{2} \mathrm{~S}$ 的 “关-开” 识别. 探针 $\mathbf{L}$ 具有斯托克位移大 $(174 \mathrm{~nm})$, 酸碱耐受性好, 对 $\mathrm{H}_{2} \mathrm{~S}$ 的识别具有抗干扰能力强、选择性好、响应速度快 (3 min 内)和检测限较低 $\left(1.18 \mu \mathrm{mol} \cdot \mathrm{L}^{-1}\right)$ 等优点, 同时, 探针 $\mathbf{L}$ 在过量 $\mathrm{H}_{2} \mathrm{~S}$ 存在下, 能够用于 $2 \leqslant \mathrm{pH} \leqslant 6.5$ 范围 内 $\mathrm{pH}$ 的检测 $\left(R^{2}=0.98764\right)$. 此外, 探针 $\mathbf{L}$ 还可以在 365 
$n m$ 紫外灯照射下直接用于 $\mathrm{H}_{2} \mathrm{~S}$ 的可视化检测. 该研究 工作在 $\mathrm{H}_{2} \mathrm{~S}$ 的检测和过量 $\mathrm{H}_{2} \mathrm{~S}$ 存在下对酸性环境中 $\mathrm{pH}$ 测试具有潜在的应用价值.

\section{3 实验部分}

\section{1 仪器与试剂}

$\mathrm{pH}$ 值用 PHS-3C 型 $\mathrm{pH}$ 计测定; IR 用 NEXUS 测定; ${ }^{1} \mathrm{H} N M R,{ }^{13} \mathrm{C}$ NMR 用 Varian Mercury plus-600 测定; 苂 光光谱用 HORIBA FluoroMax-4 测定; HRMS 用 Thermo Scientific Q Exactive 测定. 实验用水为二次蒸馏水, 二 甲亚砜为色谱纯 (J\&K 公司), 硫化钠(Alfa 公司), 其他试 剂及药品均为市售分析纯, 使用前未进行二次纯化, 实 验过程中的 $\mathrm{H}_{2} \mathrm{~S}$ 均为 $\mathrm{Na}_{2} \mathrm{~S}$ 提供.

\section{2 实验方法}

3.2.1 中间体 $N$-(2-丙炔基)-4-溴-1,8,-䒬二酰亚胺 (3) 的合成

将 2-丙炔胺 (55 mg, $10 \mathrm{mmol}$ )和 4-溴-1,8-菜二酸䣶 $(2.77 \mathrm{~g}, 10 \mathrm{mmol})$ 溶于乙二醇单甲醚 $(20 \mathrm{~mL})$ 中, 摚拌回 流 $24 \mathrm{~h}$, 冷却后, 过滤分离沉淀, 用 $\mathrm{DMF}-\mathrm{H}_{2} \mathrm{O}$ 重结晶, 得到黄色固体 $\mathbf{3}^{[34]} 2.57 \mathrm{~g}$, 产率 $81.8 \%$. m.p. 230 $232{ }^{\circ} \mathrm{C} ;{ }^{1} \mathrm{H}$ NMR (600 MHz, DMSO- $\left.d_{6}\right) \delta: 8.57$ (dd, $J=$ $25.8,7.7 \mathrm{~Hz}, 2 \mathrm{H}), 8.35(\mathrm{~d}, J=7.8 \mathrm{~Hz}, 1 \mathrm{H}), 8.22(\mathrm{~d}, J=7.7$ $\mathrm{Hz}, 1 \mathrm{H}), 8.00$ (t, $J=7.8 \mathrm{~Hz}, 1 \mathrm{H}), 4.78(\mathrm{~s}, 2 \mathrm{H}), 3.18$ (s, $1 \mathrm{H}) ;{ }^{13} \mathrm{C}$ NMR (151 MHz, DMSO- $\left.d_{6}\right) \delta: 162.6,162.5$, $133.5,132.4,131.9,131.7,130.3,130.1,129.4,128.7$, 122.8, 122.1, 79.5, 73.6 , 29.7. HRMS calcd for $\mathrm{C}_{15} \mathrm{H}_{9} \mathrm{Br}-$ $\mathrm{NO}_{2}[\mathrm{M}+\mathrm{H}]^{+}$313.9811, found 313.9811.

3.2.2 荧光探针 $N$-(2-丙炔基)-4-叠氮- 1,8 - 䓠二酰亚胺 (L) 的合成

在室温下将化合物 2 (314 mg, 1.0 mmol) 和 DMF (10 $\mathrm{mL}$ )的混合物搅拌 $30 \mathrm{~min}$ 后, 将 $\mathrm{NaN}_{3}(98 \mathrm{mg}, 1.5 \mathrm{mmol}$ ) 小心的加入上述混合物中. 将溶液在室温下摚拌过夜, 薄层色谱(TLC)检测至反应完成后，将混合物倒入 20 $\mathrm{mL}$ 蒸馏水中, 过滤沉淀并用蒸馏水洗涤. 将所得粗固 体以 $\mathrm{CH}_{2} \mathrm{Cl}_{2} / \mathrm{CH}_{3} \mathrm{OH}(V / V=100 / 1)$ 作为洗脱剂, 硅胶柱 色谱法纯化, 得到黄色固体化合物 L $940 \mathrm{mg}$, 产率 $85 \%$. m.p. $183 \sim 184{ }^{\circ} \mathrm{C} ;{ }^{1} \mathrm{H}$ NMR $\left(600 \mathrm{MHz}, \mathrm{CDCl}_{3}\right) \delta: 8.59$ $(\mathrm{d}, J=7.3 \mathrm{~Hz}, 1 \mathrm{H}), 8.53(\mathrm{~d}, J=8.0 \mathrm{~Hz}, 1 \mathrm{H}), 8.38(\mathrm{~d}, J=$ $8.4 \mathrm{~Hz}, 1 \mathrm{H}), 7.72 \sim 7.67(\mathrm{~m}, 1 \mathrm{H}), 7.40(\mathrm{~d}, J=8.0 \mathrm{~Hz}, 1 \mathrm{H})$, $4.91(\mathrm{~s}, 2 \mathrm{H}), 2.19(\mathrm{~s}, 1 \mathrm{H}) ;{ }^{13} \mathrm{C}$ NMR (151 MHz, $\left.\mathrm{CDCl}_{3}\right) \delta$ : $163.0,162.6,143.8,132.5,132.0,129.1,129.0,126.8$, 124.2, 122.1, 118.3, 114.6, 78.5, 70.6, 29.4. HRMS calcd for $\mathrm{C}_{15} \mathrm{H}_{9} \mathrm{O}_{2} \mathrm{~N}_{4} \mathrm{Na}[\mathrm{M}+\mathrm{H}+\mathrm{Na}]^{+}$300.0623, found 300.0534 .
3.2.3 $\mathrm{N}$-(2-丙炔基)-4-氨基-1,8-䒬二酰亚胺 $\left(\mathbf{L}-\mathbf{N H}_{\mathbf{2}}\right)$ 的合成

将化合物 $\mathbf{L}(276 \mathrm{mg}, 1.0 \mathrm{mmol})$ 和 $\mathrm{CH}_{3} \mathrm{CN} / \mathrm{H}_{2} \mathrm{O}$ $(V: V=1: 1,30 \mathrm{~mL})$ 混合物均匀后, 小心加入 $\mathrm{Na}_{2} \mathrm{~S}$ (780 mg, $10 \mathrm{mmol})$. 在室温下搅拌反应 $5 \mathrm{~h}$, 薄层色谱 (TLC)监测至反应完成后, 将反应混合物倒入 $50 \mathrm{~mL}$ 蒸 馏水, 用 $\mathrm{CH}_{2} \mathrm{Cl}_{2}$ 萃取, 无水 $\mathrm{Mg}_{2} \mathrm{SO}_{4}$ 干燥, 粗产物用硅 胶柱色谱法纯化, 以 $\mathrm{CH}_{2} \mathrm{Cl}_{2} / \mathrm{CH}_{3} \mathrm{OH}(V: V=100: 1)$ 为 洗脱剂, 得到浅黄色固体 $\mathbf{L}-\mathbf{N H}_{\mathbf{2}} 198 \mathrm{mg}$, 产率 79\%. m.p. $>300{ }^{\circ} \mathrm{C} ;{ }^{1} \mathrm{H}$ NMR $\left(600 \mathrm{MHz}, \mathrm{DMSO}-d_{6}\right) \delta: 8.64(\mathrm{~d}$, $J=8.4,1.2 \mathrm{~Hz}, 1 \mathrm{H}), 8.44(\mathrm{~d}, J=7.3,1 \mathrm{~Hz}, 1 \mathrm{H}), 8.21(\mathrm{~d}$, $J=8.4 \mathrm{~Hz}, 1 \mathrm{H}), 7.66(\mathrm{~d}, J=8.4,1 \mathrm{H}), 7.53(\mathrm{~s}, 2 \mathrm{H}), 6.86(\mathrm{~d}$, $J=8.4 \mathrm{~Hz}, 1 \mathrm{H}), 4.74(\mathrm{~d}, J=2.5 \mathrm{~Hz}, 2 \mathrm{H}), 3.08(\mathrm{t}, J=2.4$ $\mathrm{Hz}, 1 \mathrm{H}) ;{ }^{13} \mathrm{C}$ NMR $\left(151 \mathrm{MHz}\right.$, DMSO- $\left.d_{6}\right) \delta: 163.5,162.4$, $153.5,134.7,131.7,130.2,130.1,124.4,121.8,119.8$, 108.7, 107.4, 80.4, 72.8, 29.1. HRMS calcd for $\mathrm{C}_{15} \mathrm{H}_{10^{-}}$ $\mathrm{O}_{2} \mathrm{~N}_{4}[\mathrm{M}+\mathrm{H}]^{+}$251.0815, found 251.0816.

\section{2 .4 光谱测试}

将 $\mathbf{L}$ 溶解在 $\mathrm{CH}_{3} \mathrm{CN}$ 中得到 $1 \mathrm{mmol} \cdot \mathrm{L}^{-1}$ 储备溶液, 避光保存. 将相应待分析物 $\left(\mathrm{Ca}\left(\mathrm{ClO}_{4}\right)_{2}, \mathrm{Mg}\left(\mathrm{ClO}_{4}\right)_{2}\right.$, $\mathrm{NaClO}_{4}, \mathrm{KClO}_{4}, \mathrm{LiCl}, \mathrm{Al}\left(\mathrm{NO}_{3}\right)_{3}, \mathrm{NaF}, \mathrm{NaCl}, \mathrm{NaBr}, \mathrm{NaI}$, $\mathrm{NaClO}_{3}, \mathrm{Na}_{3} \mathrm{PO}_{4}, \mathrm{Na}_{2} \mathrm{CO}_{3}, \mathrm{NaHCO}_{3}, \mathrm{NaNO}_{3}, \mathrm{NaNO}_{2}$, $\mathrm{NaOAc}, \mathrm{Na}_{2} \mathrm{SO}_{4}, \mathrm{NaCN}, \mathrm{NaHSO}_{3}, \mathrm{Na}_{2} \mathrm{SO}_{3}, \mathrm{NaClO}$, $\mathrm{KSCN}, \mathrm{H}_{2} \mathrm{O}_{2}$, Sar, Ala, Glu, AA, Phe, GSH, Cys)溶解于蒸 馏水中, 制备分析物储备溶液. 按标准方法制备磷酸盐 缓冲液 (PBS). 使用 $\mathrm{CH}_{3} \mathrm{CN} / \mathrm{PBS}(V: V=1: 1,20$ $\left.\mathrm{mmol} \cdot \mathrm{L}^{-1}, \mathrm{pH}=7.4\right)$ 将新鲜储备溶液稀释至所需浓度. 实验过程测定 $\mathrm{H}_{2} \mathrm{~S}$ 时，将荧光探针 $\mathbf{L}$ 用 $\mathrm{CH}_{3} \mathrm{CN}$ 稀释成 $0.2 \mathrm{mmol} \cdot \mathrm{L}^{-1}$ 的溶液, 移取 $30 \mu \mathrm{L}\left(0.2 \mathrm{mmol} \cdot \mathrm{L}^{-1}\right) \mathbf{L}$ 溶液 至比色皿, 然后加入 $2.970 \mathrm{~mL} \mathrm{CH}_{3} \mathrm{CN} / \mathrm{PBS}(V: V=1$ : $1,20 \mathrm{mmol} \cdot \mathrm{L}^{-1}, \mathrm{pH}=7.4$ ) 缓冲溶液以获得最终浓度为 1 $\mu \mathrm{mol} \cdot \mathrm{L}^{-1}$ 的测试液. 然后, 将 $0 \sim 20 \mu \mathrm{mol} \cdot \mathrm{L}^{-1} \mathrm{H}_{2} \mathrm{~S}$ 溶液 滴加到上述溶液中, 混合 $5 \mathrm{~min}$ 后在室温下进行苂光光 谱测定. 所有检测实验均在 $\mathrm{CH}_{3} \mathrm{CN} / \mathrm{PBS}(V: V=1: 1$, $\left.20 \mathrm{mmol} \cdot \mathrm{L}^{-1}, \mathrm{pH}=7.4\right)$ 中进行. 苂光测量的激发波长为 $420 \mathrm{~nm}$ ，激发和发射的狭缝宽度均为 $3 \mathrm{~nm}$.

\subsection{5 $\mathrm{pH}$ 检测}

不同 $\mathrm{pH}$ 的 $\mathrm{PBS}$ 缓冲溶液均用稀 $\mathrm{HCl}$ 溶液和稀 $\mathrm{NaOH}$ 溶液调节得到. 分别向一组试管中加入 $\mathrm{H}_{2} \mathrm{~S}(9$ $\mu \mathrm{L}, 10 \mathrm{mmol} \cdot \mathrm{L}^{-1}$ ) 和 $2.976 \mathrm{~mL}$ 不同 $\mathrm{pH}$ 值的 $\mathrm{CH}_{3} \mathrm{CN} / \mathrm{PBS}$ 混合溶液 $\left(10 \mathrm{mmol} \cdot \mathrm{L}^{-1}, V: V=1: 1, \mathrm{pH}=2.0,2.5,3.0\right.$, $3.5,4.0,4.5,5.0,5.5,6.0,6.5,7.0,7.5,8.0,8.5,9.0,9.5$, $10,11,12,12.6)$, 混合均匀后再加入 $\mathbf{L}(15 \mu \mathrm{L}, 0.2$ $\left.\mathrm{mmol} \cdot \mathrm{L}^{-1}\right)$, 每根试管的溶液总体积为 $3 \mathrm{~mL}$, 苂光探针 浓度为 $1 \mu \mathrm{mol} \cdot \mathrm{L}^{-1}$, 在室温下反应 $5 \mathrm{~min}$, 测定其苂光光 
谱.

\subsection{6 理论计算}

采用密度泛函理论(DFT) 对苂光探针分子 $\mathbf{L}$ 和 $\mathbf{L}_{-} \mathbf{N H}_{2}$ 分别进行了几何优化和量子化学计算, 并在 Gaussian 09 程序采用 DFT/B3LYP/6-31G (d,p)基组进行 了结构优化. 利用含时密度泛函理论(TD-DFT), 在相同 的基组下, 得到了最高占据分子轨道(HOMO)和最低未 占据分子轨道(LUMO)的能级.

\section{辅助材料(Supporting Information) 化合物的红外光} 谱、高分辨质谱、核磁共振氢谱和碳谱谱图. 这些材料 可以免费从本刊网站(http://sioc-journal.cn/)上下载.

\section{References}

[1] Sjaastad, O.; Bakketeig, L. S. Cephalalgia 2006, 26, 466.

[2] Szabó, C. Nat. Rev. Drug Discovery 2007, 6, 917.

[3] Kaushik, R.; Ghosh, A.; Jose D. A. Coord. Chem. Rev. 2017, 347, 141.

[4] Zhou, C.; Qiu, B.; Zeng, Y.; Chen, J. P.; Yu, T. J.; Li, Y. Chin. J. Org. Chem. 2017, 37, 92 (in Chinese). (周婵, 邱波，曾毅，陈金平，于天君，李姫，有机化学, 2017, 37, 92.)

[5] Sun, L.; Wu, Y.; Chen, J.; Zhong, J.; Zeng, F.; Wu, S. Theranostics 2019, 9, 77 .

[6] Abe, K.; Kimura, H. J. Neurosci. 1996, 16, 1066.

[7] Yang, G.; Wu, L.; Jiang, B.; Yang, W.; Qi, J.; Cao, K.; Zhang, S. Science 2008, 322, 587.

[8] Lavu, M.; Bhushan, S.; Lefer, D. J. Clin. Sci. 2011, 120, 219.

[9] de Sousa, M. C.; Vieira, R. B.; Dos Santos, D. S.; Carvalho, C. A.; Camargo, S. E.; Mancini, M. N.; de Oliveira, L. D. Arch. Oral Biol. 2015, 60, 600 .

[10] Shi, W.; Pan, M.; Qiang, H.; Qiu, Q.; Huang, W.; Lin, H.; Qian, H.; Ge, L. Chem. Biol. Drug Des. 2017, 90, 167.

[11] Li, X.; Liu, Q.; Ye, S.; Wang, S.; Li, K.; Lv, G.; Peng, Y.; Qiu, L.; Lin, J. Chem. Biol. Drug Des. 2019, 94, 1494.

[12] Eto, K.; Asada, T.; Arima, K.; Makifuchi, T.; Kimura, H. Biochem. Biophys. Res. Commun. 2002, 293, 1485.
[13] Wang, H.; Yang, D.; Tan, R.; Zhou, Z. J.; Xu, R.; Zhang, J. F. Zhou, Y. Sens. Actuators, B 2017, 247, 883.

[14] Shi, D. T.; Zhou, D.; Zang, Y.; Li J.; Chen, G. R.; James, T. D.; He, X. P.; Tian, H. Chem. Commun. 2015, 51, 3653.

[15] Chen, S.; Ma, C.; Wang, D.-E.; Han, X.; Zhang, L.; Wang, J. Anal. Methods 2015, 7, 7646.

[16] Feng, X.; Zhang, T.; Liu, J.-T.; Miao, J.-Y.; Zhao, B.-X. Chem. Commun. 2016, 52, 3131.

[17] Cao, D.; Liu, Z.; Verwilst, P.; Koo, S.; Jangjili, P.; Kim, J. S.; Lin, W. Chem. Rev. 2019, 119, 10403.

[18] Peng, H.; Cheng, Y.; Dai, C.; King, A. L.; Predmore, B. L.; Lefer, D. J.; Wang, B. Angew. Chem., Int. Ed. 2011, 50, 9672.

[19] He, P.; Tang, L.-J; Zhong, K.-L.; Hou, S.-H.; Yan, X.-M. Chin. J. Org. Chem. 2017, 37, 423 (in Chinese).

(何平, 汤立军, 钟克利, 侯淑华, 燕小梅, 有机化学, 2017, 37, 423.)

[20] Wang, H.; Wu, X.-M.; Yang, S.-X.; Tian, H.-Y.; Liu, Y.-G.; Sun, B.-G. Dyes Pigm. 2019, 160, 757.

[21] Renault, K.; Renard, P.-Y.; Sabot, C. New J. Chem. 2017, 41, 10432.

[22] Li, H.; Feng, X.; Guo, Y.; Chen, D.; Li, R.; Ren, X. Sci. Rep. 2014, 4, 4366.

[23] Jun, M. E.; Roy, B.; Ahn, K. H. Chem. Commun. 2011, 47, 7583.

[24] Chen, H.; Gong, X.; Liu, X.; Li, Z.; Zhang, J.; Yang, X.-F. Sens. Actuators, B 2019, 281, 542.

[25] Chen, Y.; Zhu, C.; Yang, Z. Chen, J.; He, Y.; Jiao, Y. Angew. Chem., Int. Ed. 2013, 52, 1688.

[26] Lippert, R.; New, E. L.; Chang, C. J. J. Am. Chem. Soc. 2011, 133, 10078.

[27] Lippert, A. R. J. Inorg. Biochem. 2014, 133, 136.

[28] Platt, F. M. Nature. 2014, 510, 68.

[29] Liu, X.; Su, Y.; Tian, H.; Yang, L.; Zhang, H.; Song, X.; Foley, J. W. Anal. Chem. 2017, 89, 7038.

[30] Xue, Z.; Zhao, H.; Liu, J.; Han, J.; Han, S. ACS Sens. 2017, 2, 436.

[31] Sun, T.; Xia, L.; Huang, J.; Gu, Y.; Wang, P. Talanta 2018, 187, 295.

[32] Fan, F.; Jing, J.; Chen, X. Chin. J. Org. Chem. 2014, 34, 2178 (in Chinese). (范方禄，靖金球，陈雪梅，有机化学, 2014, 34, 2178.)

[33] Peng, S.; Zhong, T.; Guo, T.; Shu, D.; Meng, D.; Liu, H.; Guo, D. New J. Chem. 2018, 42, 5185.

[34] Pancholi, J.; Hodson, D. J.; Jobe, K.; Rutter, G. A.; Goldup, S. M.; Watkinson, M. Chem. Sci. 2014, 5, 3528.

(Li, L.; Fan, Y.) 